\title{
PRENATAL DEVELOPMENT OF THE MESONEPHROS IN RABBITS
}

\author{
W. GABER \\ Department of Anatomy, Histology and Embryology, Faculty of Veterinary Medicine, \\ Assiut University, Assiut, Egypt
}

Received: 28 June 2017; $\quad$ Accepted: 30 July 2017

\begin{abstract}
The developmental changes of the rabbit mesonephros during the prenatal life were studied in 31 rabbits at embryonic days (E) 10-24 for light microscopical investigation. Generally, the mesonephros consists of mesonephric duct, tubules and glomeruli. At E 10, the mesonephros includes the mesonephric duct and tubules only as the glomerulus appears at E 11 where, the mesonephric corpuscles show different stages of development. At E 14\&15, the mesonephros reaches its maximum size, degree of differentiation and function where, the cells of the secretory tubules show intense P.A.S. activity at their luminal border. The rabbit mesonephros begins to degenerate at E 16 while, the metanephros is early developed at this age and so, they are not overlapped in capability of function. Disappearance of the mesonephros starts at E 20 and it could not be observed at E 24. In conclusion, the rabbit mesonephros is large, present in fully developed state for about 4 days (from 13 to 16 days of gestation) and begins its degeneration early at E 16.
\end{abstract}

Key words: Rabbit - Mesonephros - Prenatal - Development.

\section{INTRODUCTION}

The embryos of mammals develop a mesonephros which is functional during a part of fetal life (Arey, 1965). The development of the mesonephros has been studied by many authors; Patten (1964) in pig, Canfield (1980) in bovine, (Tiedmann and Wettstein, 1980; El-Gharbawy, 2002; Fayez et al., 2014) in rabbit, (Bareedy et al., 1982; Emara, 1989; El-Harairy et al., 1998; Aly, 2007; Osman et al., 2008) in camel, Moustafa et al. (1986) in buffalo and Sadler (2000) in human. The relative size and the duration for which the mesonephros remains functioning vary in different types of mammals (Bremer, 1916; Stanier, 1960). There is a close relationship between the size and persistence of the mesonephros and the size of the allantois (Davies, 1952). Bremer (1916) studied the histological interrelations of the mesonephros, kidney and placenta and stated that, the urinary excretion in rabbit embryos takes place at first through the mesonephros and later through the placenta. Although, Leeson and Baxter (1957) mentioned that the metanephros overlaps the mesonephros in the rabbit in capability of function. For this controversy, the present study is designed to investigate the development of

Corresponding author: Dr. W. GABER

E-mail address: wafaa.anatomy@gmail.com

Present address: Department of Anatomy, Histology and Embryology, Faculty of Veterinary Medicine, Assiut University, Assiut, Egypt the mesonephros in rabbits during different embryonic stages from its early differentiation till its degeneration and disappearance, and to clarify its size, function and persistence which might be helpful to solve this argument.

\section{MATERIALS AND METHODS}

This research was performed on 31 normal New Zealand white rabbit embryos at embryonic days (E) 10-24, which were collected from the Research Farm of Faculty of Agriculture, Assiut University, Egypt. The pregnant rabbits were sacrificed at various periods of gestation. The embryos were removed shortly after evisceration, and the crown vertebral rump length (CVRL) was measured (Table 1). The embryos were fixed in $10 \%$ neutral buffered formalin or Bouin's fluid for histological and histochemical techniques. The fixed specimens were dehydrated in graded alcohol series, cleared, embedded in paraffin and serially sectioned at 3-5 $\mu \mathrm{m}$ thick. The obtained sections were stained with the following stains: Harris haematoxylin and eosin stain (Harris, 1900) for general histological studies, Crossmon's trichrome stain (Crossmon, 1937) for identification of collagenous and muscle fibers, and Periodic Acid Schiff reaction (PAS) (McManus, 1946) for detection of glycoproteins. 
Table 1: Age, corresponding CVRL and number of used embryos.

\begin{tabular}{|c|c|c|c|c|c|c|c|c|c|c|c|}
\hline Age (day) & 10 & 11 & 12 & 13 & 14 & 15 & 16 & 18 & 20 & 23 & 24 \\
\hline CVRL (mm) & - & - & - & 28 & 34 & 39 & 43 & 49 & 54 & 80 & 88 \\
\hline Number & 4 & 3 & 4 & 3 & 3 & 4 & 2 & 4 & 2 & 1 & 1 \\
\hline
\end{tabular}

\section{RESULTS}

At E 10, the mesonephros appears as a long narrow strip with rounded cranial and caudal ends, extends on either side of the aorta. It projects into the coelom within the urogenital ridge on either side of the mesentery. It consists of mesonephric tubules medially and mesonephric duct laterally. No glomeruli could be observed. The mesonephric tubules are S-shaped and differentiated into proximal part representing the secretory tubules and distal part, the collecting ones. The secretory tubules lie medially and are larger than the collecting tubules. The lining epithelium of the secretory tubules is higher than that of the collecting ones. Some of the mesonephric tubules remain solid and others are connected with the mesonephric duct which is lined by cuboidal epithelium. The intertubular spaces are wide and filled with loose mesenchymal tissue (Figs. 1\&2).

At E 11, the mesonephric corpuscles including the glomerulus and the glomerular capsule begin to differentiate. The medial end of the secretory tubules becomes ampullated and invaginated by the glomerulus to form the glomerular capsule. The aorta gives off several branches, from its ventrolateral aspect, which invade the medial aspect of the mesonephros. Within the mesonephros, these branches divide into tufts of arterioles forming the glomerulus. The glomerular capsule is crescentic in cross section, consisting of visceral and parietal layers separated by the capsular cavity. The capsular cavity shows different degrees of its width. The parietal layer is lined by simple squamous epithelium. The visceral layer is lined by cuboidal epithelium in the cranial third of the mesonephros while in the caudal two thirds; it is lined by low columnar epithelium. This layer is directly in contact with the glomerulus. Some glomeruli contain red blood cells. At this age, the mesonephros is narrow and consists of three rows in section; medial row represents the mesonephric corpuscles, middle row represents the secretory tubules and lateral row represents the collecting tubules (Fig. 3). All components of the mesonephros show no PAS reaction.

At E 12, the mesonephros appears in the form of a long narrow band extending from the level of the first thoracic somite to the sixth lumbar somite (Fig. 4). The cranial part of the mesonephros is separated from the stomach by the dorsal mesogastrium on the left side and from the liver by the celom on the right side. The posterior cardinal vein could be observed dorsal to the mesonephros. It is relatively large and gives off blood sinusoids to the intertubular mesenchyme (Fig. $5)$. The glomeruli of the cranial corpuscles become larger and containlarge number of red blood cells. The parietal layer of the glomerular capsule remains flattened. The visceral layer in the cranial two thirds of the mesonephros is lined by cuboidal epithelium while, that of the caudal third remains lined by low columnar epithelium. The secretory tubules become coiled consequently; they appear 2-3 rows in section. There is a slight PAS-positive material in the luminal border. The intertubular mesenchyme remains wide, loose and less vascular (Fig. 6).

At E 13, the mesonephros becomes larger therefore; its cranial part is related ventrally to the stomach on the left side and the liver on the right side. Caudally, it is separated from the intestine by the celom and ventromedially, it is related to the gonad (Fig. 7). The mesonephric corpuscle shows vascular pole and urinary pole. The blood vessels could be observed passing through the vascular pole. The urinary pole connects the capsular cavity with the secretory tubule through the neck. The glomeruli become larger and numerous. The visceral layer of the glomerular capsule becomes lined by cuboidal epithelium throughout the whole length of the mesonephros. The flattened epithelium of the parietal layer thickens into cuboidal then low columnar epithelium for about 4-8 cells in the neck to continue with the low columnar epithelium of the secretory tubule (Fig. 8). The secretory and collecting tubules could be easily distinguished morphologically and histochemically. 
The secretory tubules are larger, thicker and lighter than the collecting ones. They are lined by more eosinophilic low columnar epithelium which shows a moderate PAS activity in the luminal cytoplasm while, the collecting tubules are lined by cuboidal epithelium with no PAS activity. Both types of tubules become more coiled. Although the mesonephric corpuscles are medially located and the collecting tubules are laterally located with the secretory tubules in the middle, at this age, some of these tubules were observed between the corpuscles and some corpuscles are intermingled with the secretory tubules. The intertubular mesenchyme becomes narrow, dense and more vascular as, the blood sinusoids from the posterior cardinal vein increase (Fig. 9).

At E 14, the caudal end of the mesonephros is related dorsally to the metanephros and ventrally to the intestine. The mesonephros becomes well defined and covered by flattened celomatic epitheliumwith moderate mesenchymal layer subepithelial. The mesonephric tubules become more coiled and extend more dorsally than the level of the glomeruli and the mesonephric duct. Almost all of the mesonephric tubules as well as the mesonephric duct contain structureless material within their lumen (Fig. 10).

At E 15, the mesonephros is related ventrally to the liver on the right side and the stomach on the left and laterally to the liver. Just caudal to its middle, the mesonephros is related dorsomedially to the early developing metanephros and ventromedially to the gonad. The right mesonephros is situated somewhat cranial to the left one. In transverse sections, cranially, only the right mesonephros could be observed but somewhat caudally, the right mesonephros with the gonad are opposite to the cranial end of the left mesonephros (Figs. 11a\&b). The mesonephros becomes larger. All the mesonephric corpuscles and tubules are fully developed. The glomeruli occupy about the medial half of the mesonephros. The tubules and the glomeruli are very closely related as the intertubular mesenchyme becomes very scanty and the blood sinusoids become very narrow. The epithelium of the visceral layer of the glomerular capsule becomes fullydifferentiated. An extremely thin, flange-like process extends from one side of the cell while, the remainder of the cell including the nucleus retain its cuboidal shape (Fig. 12). The secretory tubules show intense PAS-positive reaction in the luminal border of their lining epithelium while, the collecting ones show no reaction (Fig. 13).

At E 16, the cranial end of the mesonephros is degenerated otherwise, it is fully developed. The glomeruli of the degenerated part become small, packed with blood cells and the parietal layer of the glomerular capsule becomes cuboidal with thick basement membrane. The mesonephric tubules in this region become collapsed and show loss of continuity. The intertubular spaces become wider and are filled with connective tissue. At its middle, the mesonephros is related dorsally to the metanephros and ventrally to the gonad (Figs. 14-16).

At E 18, the cranial third of the mesonephros shows degeneration but the caudal two thirds remain active (Fig. 17).

At E 20, the cranial part of the mesonephros disappears while the remaining part degenerates (Fig. 18). In the degenerated tubules, there is still some PAS activity.

At E 23, all the mesonephros disappears except a very small degenerated part remains just cranial to the pelvic inlet (Fig. 19).

At E 24, no remnants of the mesonephros could be observed. 


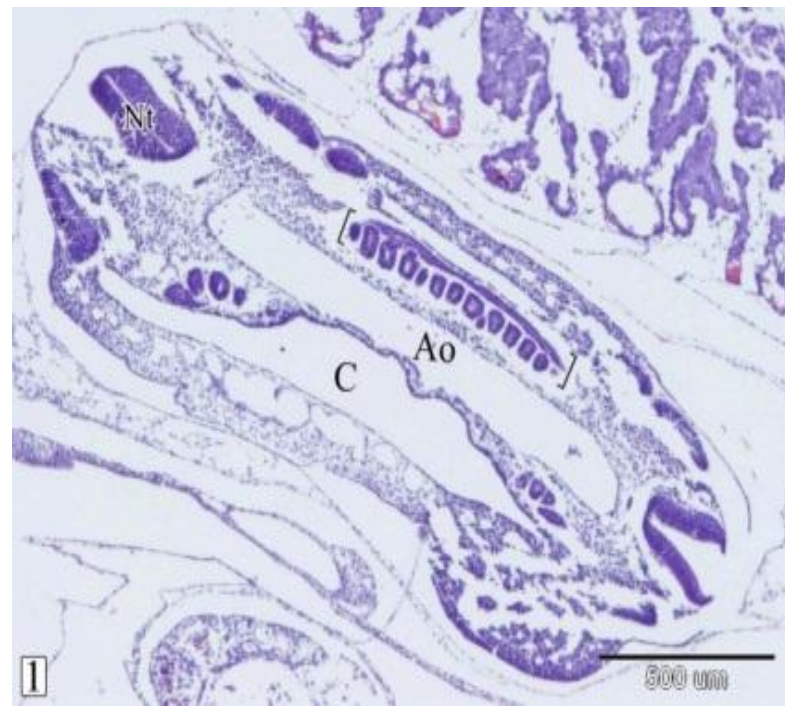

Fig. 1. Frontal section in a rabbit embryo at E 10 showing the mesonephros (in brackets) on either side of the aorta (Ao). Celom (C) and neural tube (Nt). (H\&E, X 40).

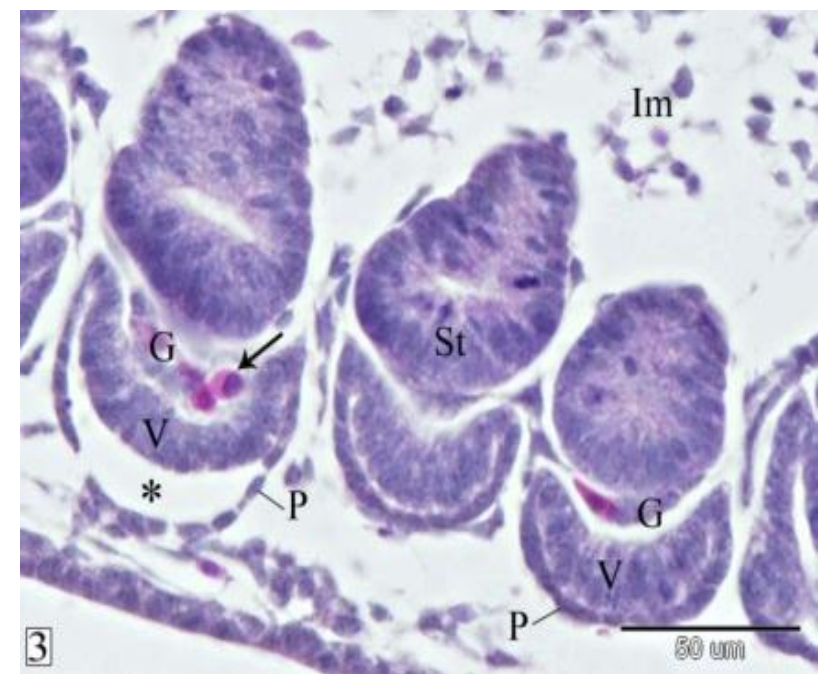

Fig. 3. Sagittal section in a rabbit embryo at E 11 showing the differentiation of the mesonephric corpuscles. Glomerulus (G), red blood cells (arrow), capsular cavity $(*)$, visceral layer $(\mathrm{V})$, parietal layer $(\mathrm{P})$, secretory tubule $(\mathrm{St})$ and intertubular mesenchyme (Im). (H\&E, X 400).

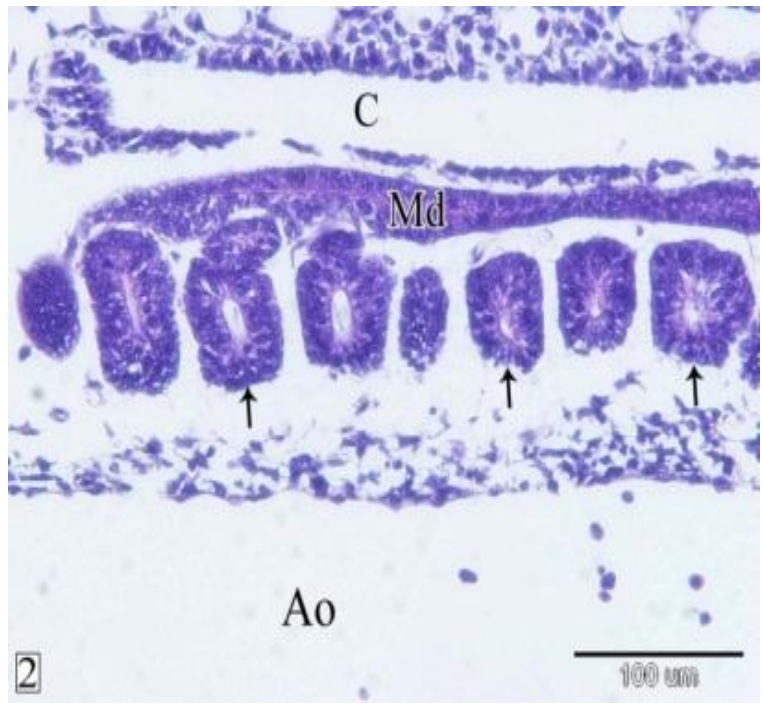

Fig. 2. Frontal section in a rabbit embryo at E 10 showing the mesonephros consists of mesonephric tubules (arrows) medially and mesonephric duct (Md) laterally. Notice, some tubules are connected with the duct. Aorta (Ao) and celom (C). (H\&E, X 200).

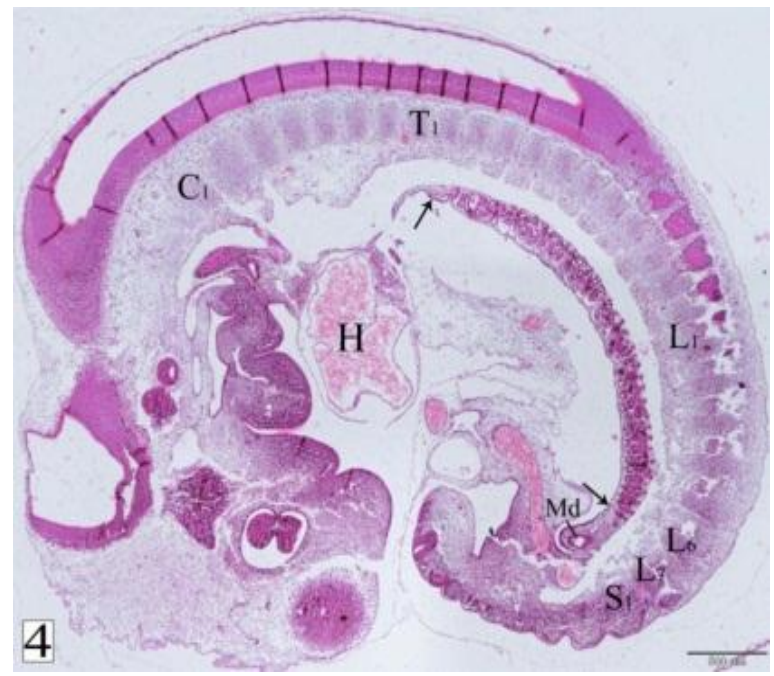

Fig. 4. Sagittal section in a rabbit embryo at E 12 showing the extension of the mesonephros (between arrows) from the level of the first thoracic somite $\left(\mathrm{T}_{1}\right)$ to the sixth lumbar somite $\left(\mathrm{L}_{6}\right)$. Mesonephric duct $(\mathrm{Md})$ and heart $(\mathrm{H})$. (H\&E, X 40). 

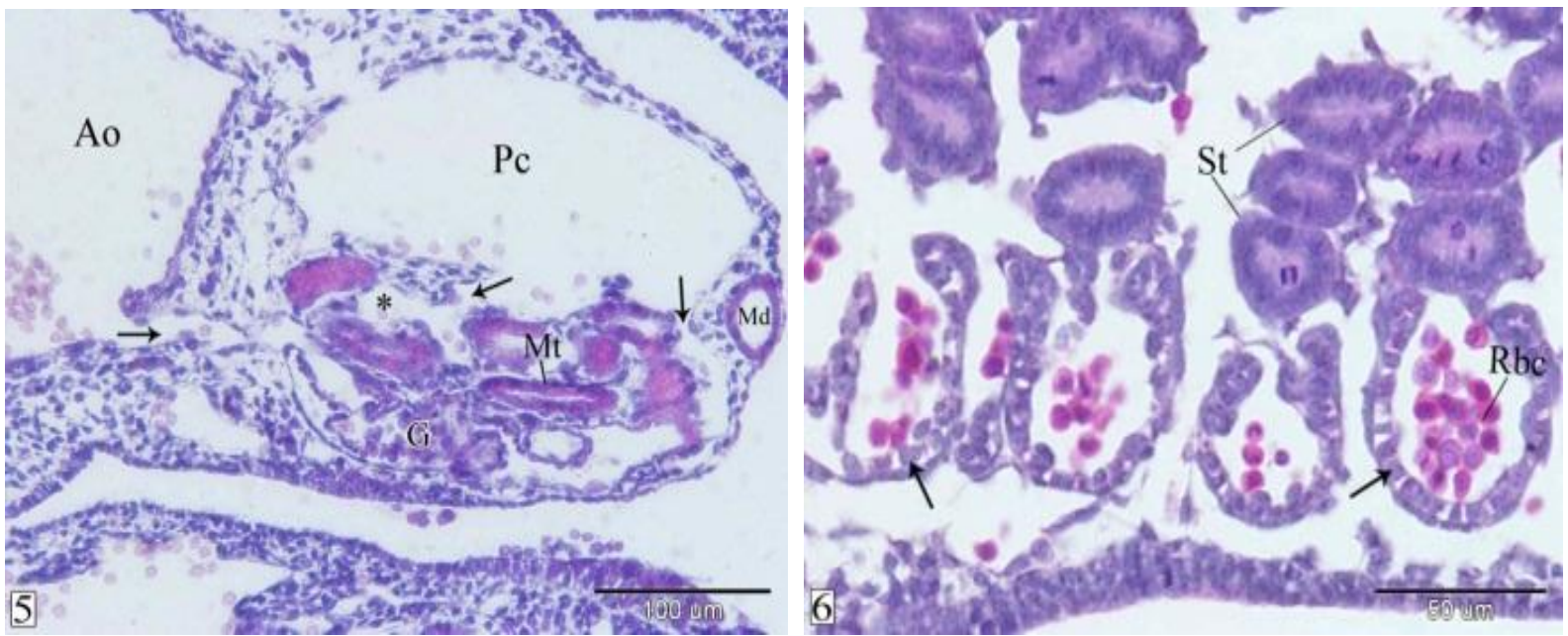

Fig. 5. Transverse section in a rabbit embryo at E 12 showing the aorta (Ao) and posterior cardinal vein (Pc) give off branches (arrows) to the mesonephros. Glomerulus (G), mesonephric tubules (Mt), mesonephric duct (Md) and blood sinusoid (*). (H\&E, X 400).

X 200).

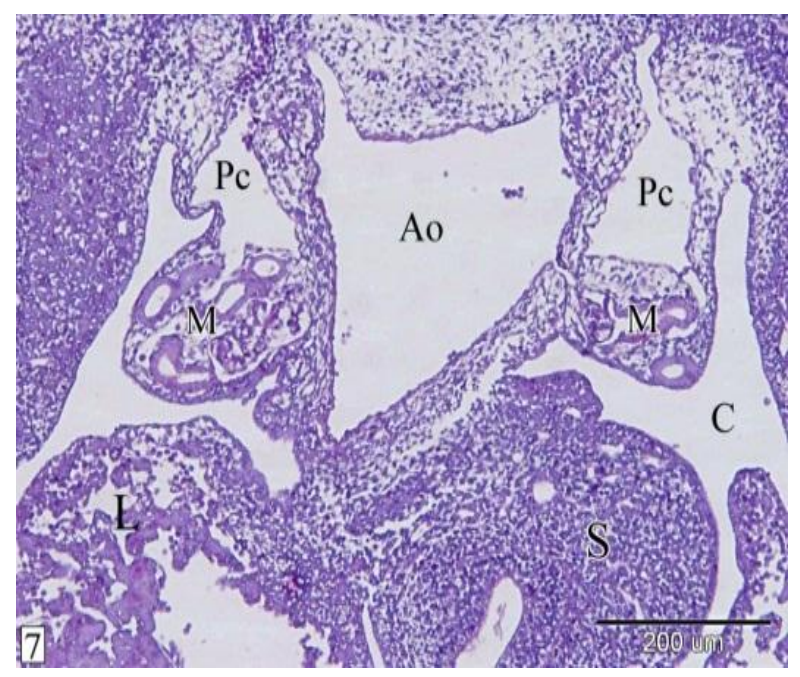

Fig. 7. Transverse section in a rabbit embryo at E 13 showing the relation of the mesonephros (M) to the surrounding structures. Liver (L), stomach (S), aorta (Ao), posterior cardinal vein (Pc) and celom (C). (H\&E, $\mathrm{X} 100)$.
Fig. 6. Sagittal section in a rabbit embryo at E 12 showing the cranial part of the mesonephros becomes well developed. Secretory tubules (St), cuboidal epithelium (arrows) and red blood cells (Rbc). (H\&E,

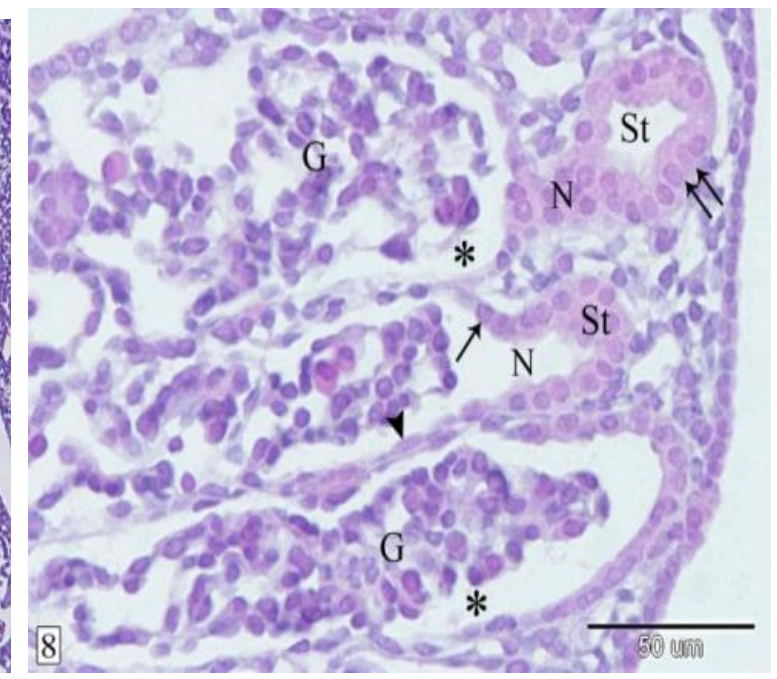

Fig. 8. Transverse section in a rabbit embryo at E 13 showing the lining epithelium of the neck $(\mathrm{N})$ which connects the glomerular capsule with the secretory tubule (St). Glomerular cavity (*), flattened epithelium (arrow head), cuboidal cells (arrow), low columnar epithelium (double arrow) and glomerulus (G). (H\&E, X 400). 

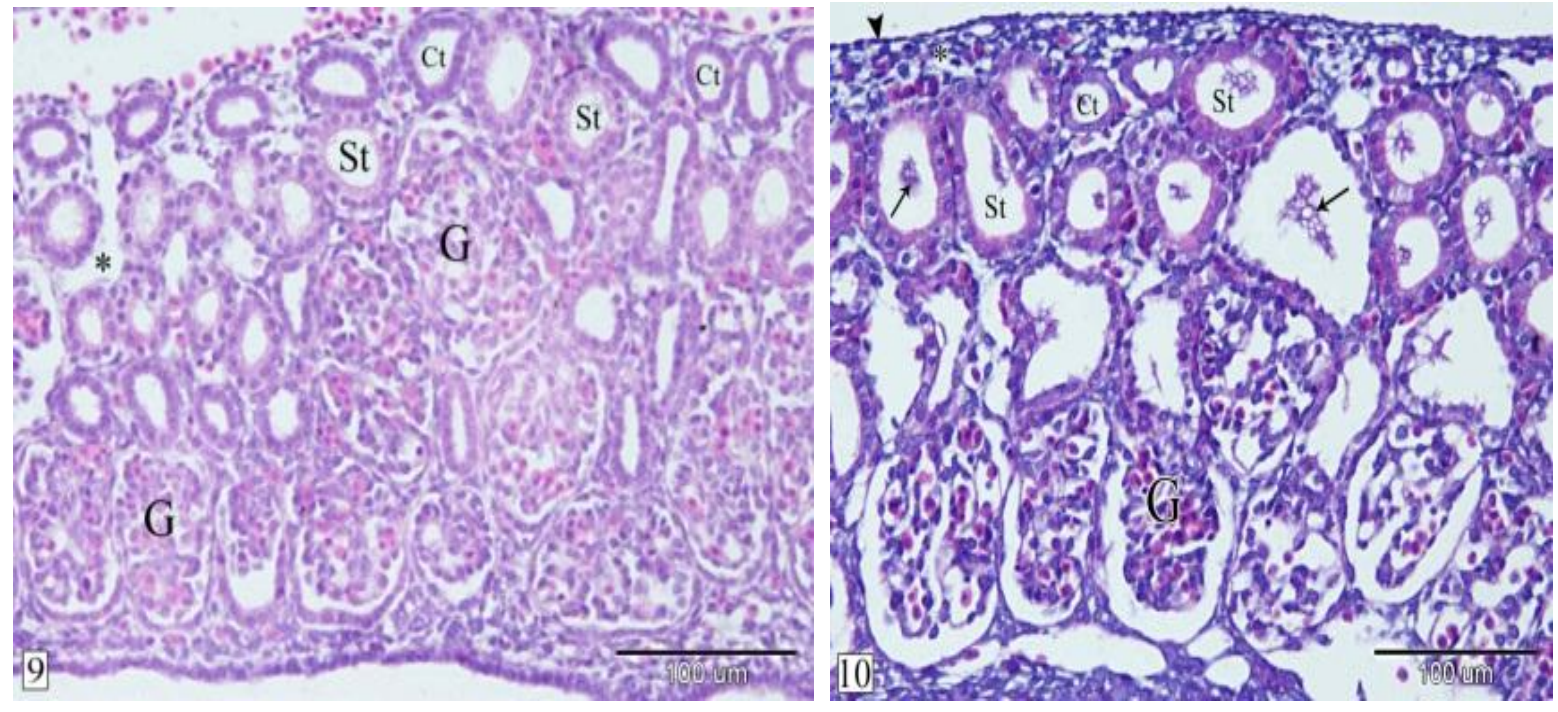

Fig. 9. Sagittal section in a rabbit embryo at E 13 showing the structure of the mesonephros. Glomerulus $(\mathrm{G})$, secretory tubule $(\mathrm{St})$, collecting tubule $(\mathrm{Ct})$ and blood sinusoids (*). (H\&E, X 200).

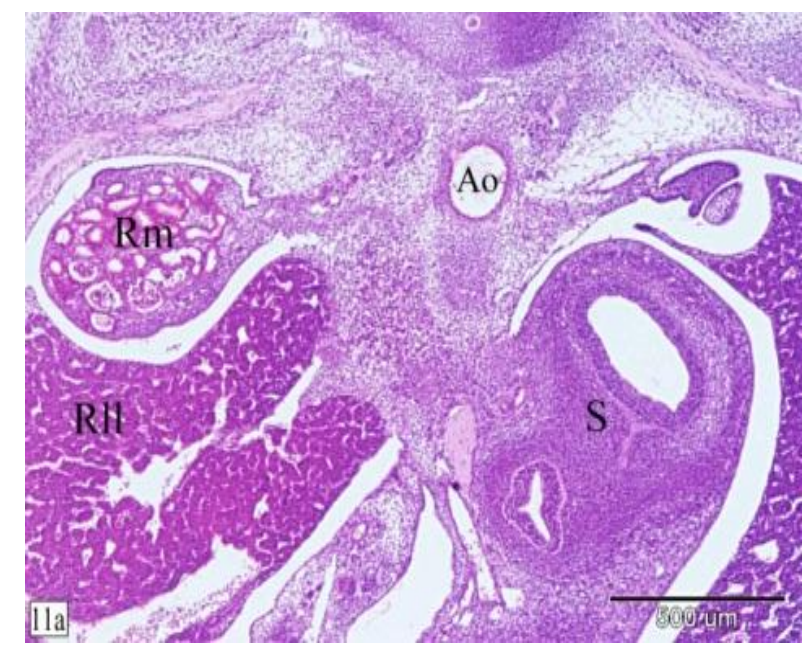

Fig. 10. Sagittal section in a rabbit embryo at E 14 showing the mesonephric tubules contain structureless material within the lumen (arrows). Secretory tubule (St), collecting tubule $(\mathrm{Ct})$, glomerulus $(\mathrm{G})$, celomatic epithelium (arrow head) and subepithelial mesenchyme (*). (H\&E, X 200).

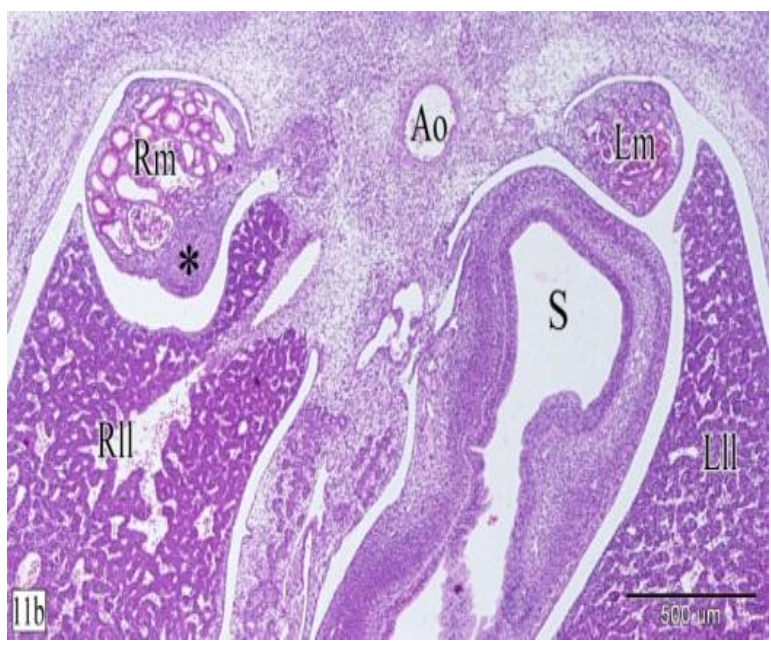

Figs. 11a\&b. Transverse sections in a rabbit embryo at E 15 showing the right mesonephros (Rm) is situated somewhat cranial to the left mesonephros (Lm). Right lobe of liver (Rll), left lobe of liver (Lll), stomach (S), gonad (*) and aorta (Ao). (11a is a more cranial level than 11b) (H\&E, X 40). 


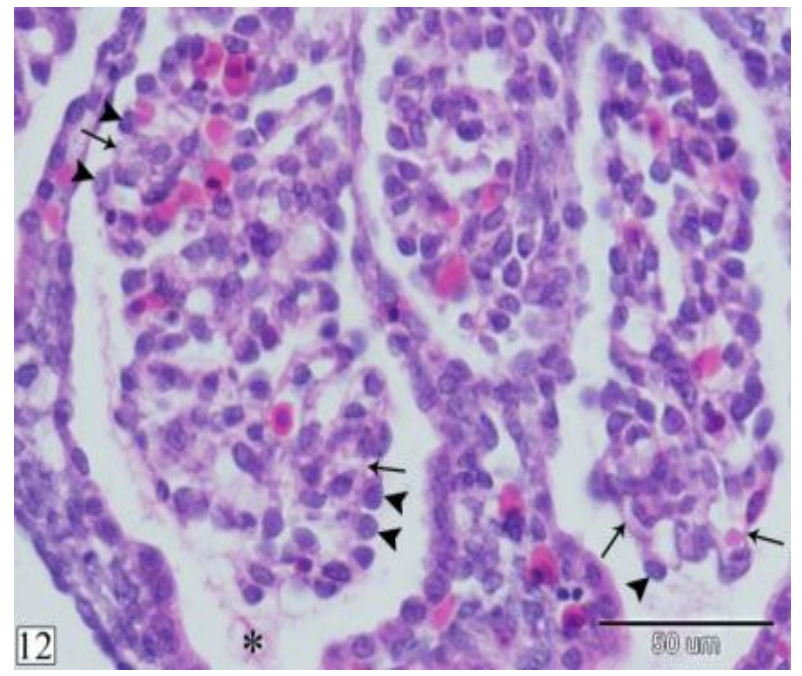

Fig. 12. Sagittal section in a rabbit embryo at E 15 showing the fully differentiated epithelium of the visceral layer of the glomerular capsule. Cuboidal cells (arrow heads), flange-like process (arrows) and glomerular cavity (*). (H\&E, X 400).

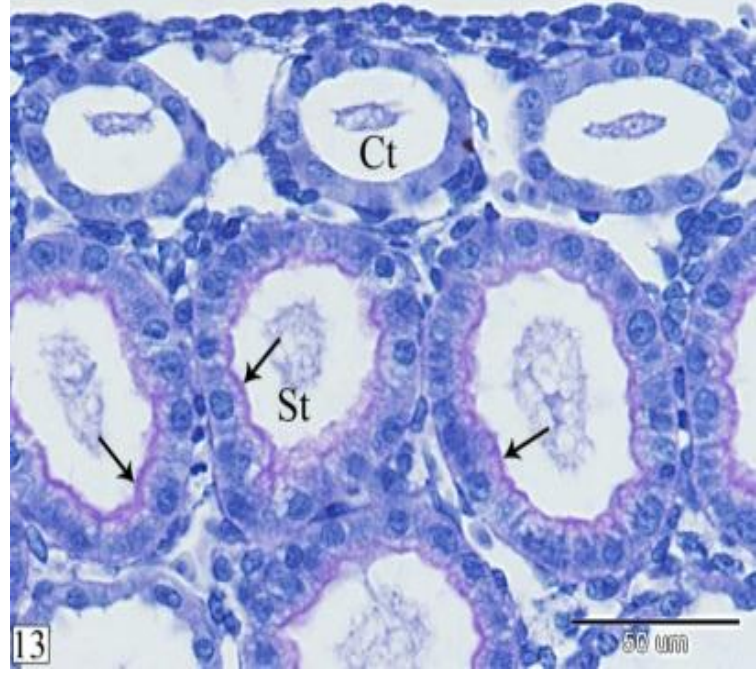

Fig. 13. Sagittal section in a rabbit embryo at E 15 showing the secretory tubules (St) have intense PASpositive reaction (arrows) while, the collecting tubules $(\mathrm{Ct})$ show no reaction. (PAS-Haematoxylin, $\mathrm{X} 400)$.

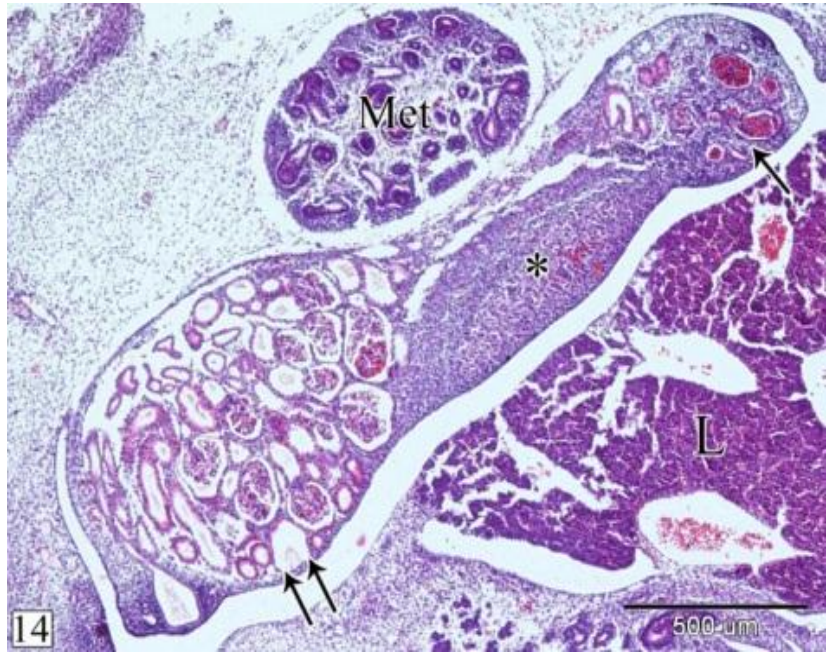

Fig. 14. Sagittal section in a rabbit embryo at E 16 showing the cranial end (arrow) of the mesonephros is degenerated while the caudal part (double arrow) is fully developed. Metanephros (Met), gonad (*) and liver (L). (H\&E, X 40).

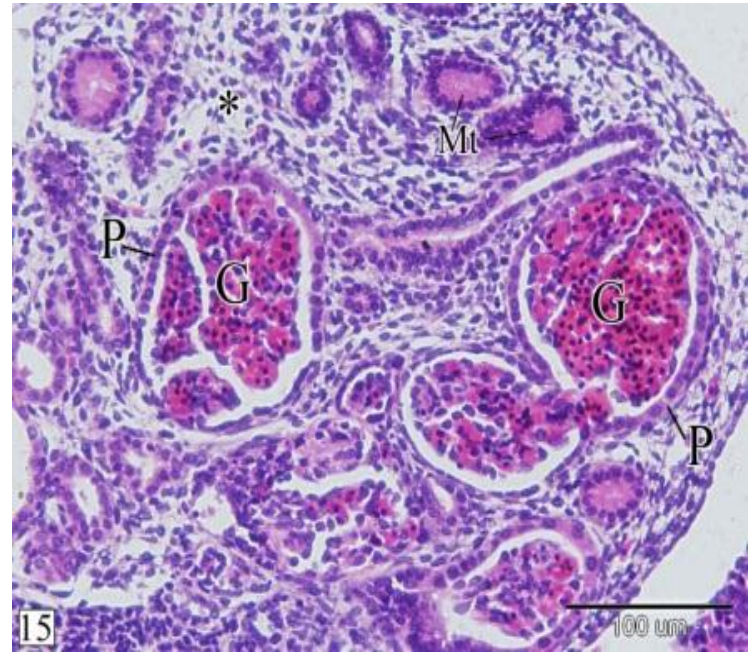

Fig. 15. Sagittal section in a rabbit embryo at E 16 showing the degenerated cranial end of the mesonephros. Glomerulus (G), parietal layer of glomerular capsule $(\mathrm{P})$, mesonephric tubules $(\mathrm{Mt})$ and intertubular connective tissue (*). (H\&E, X 200). 

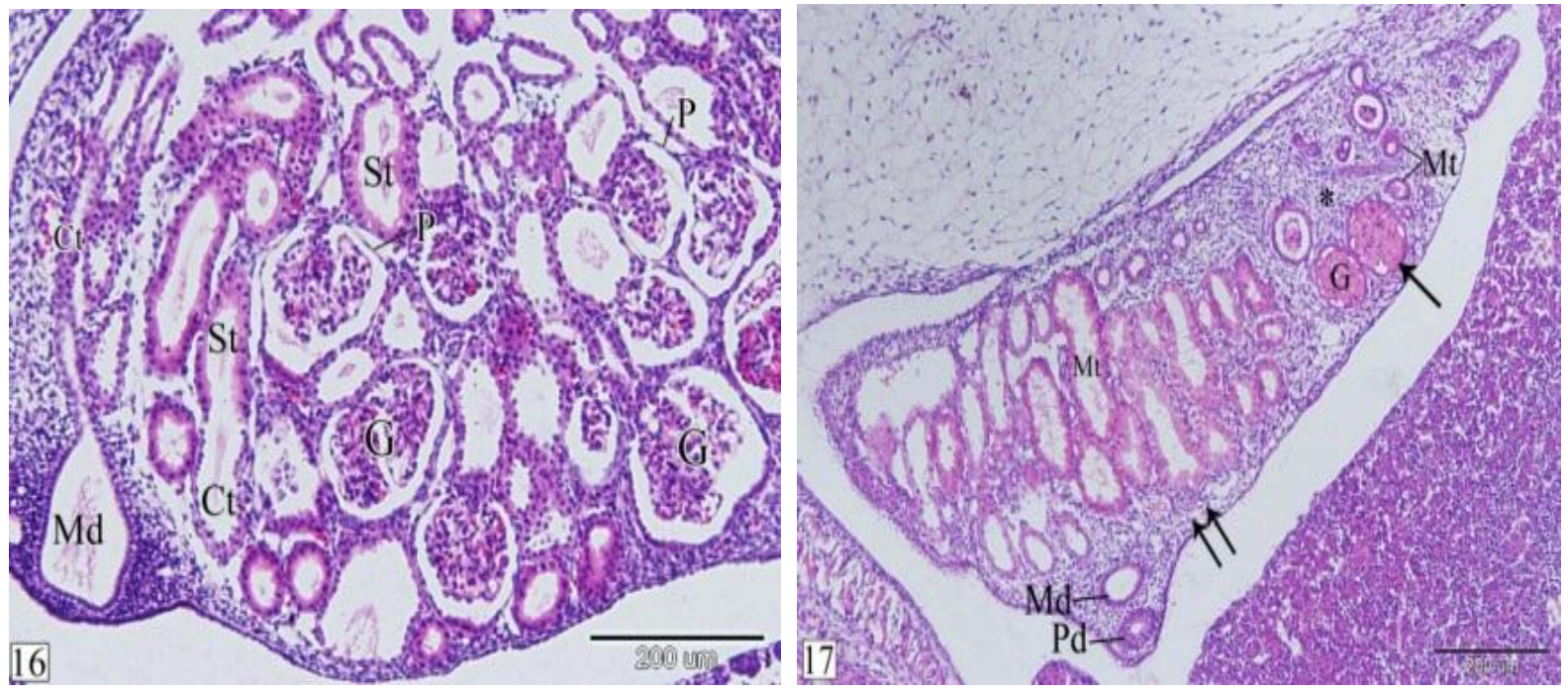

Fig. 16. Sagittal section in a rabbit embryo at E 16 showing the fully developed caudal part of the mesonephros. Glomerulus (G), parietal layer of glomerular capsule (P), secretory tubules (St), collecting tubules $(\mathrm{Ct})$ and mesonephric duct $(\mathrm{Md})$. (H\&E, X 100).
Fig. 17. Sagittal section in a rabbit embryo at E 18 showing the cranial third of the mesonephros is degenerated (arrow) but the caudal two thirds remain active (double arrow). Glomerulus (G), mesonephric tubules (Mt), intertubular connective tissue (*), mesonephric duct $(\mathrm{Md})$ and paramesonephric duct (Pd). (H\&E, X 100).

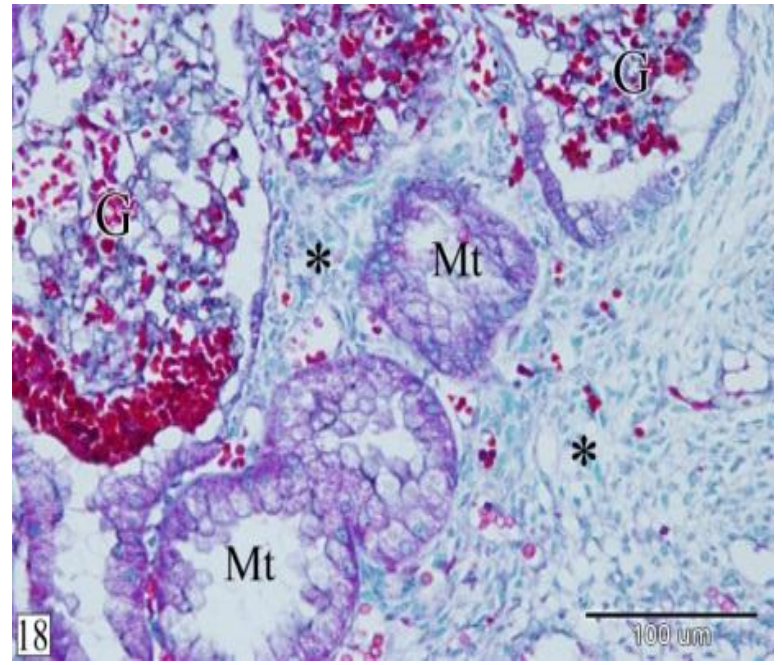

Fig. 18. Sagittal section in a rabbit embryo at E 20 showing the remaining degenerated caudal part of the mesonephros. Glomerulus $(\mathrm{G})$, mesonephric tubules (Mt), intertubular connective tissue $(*)$. (Crossmon's trichrome, X 200).

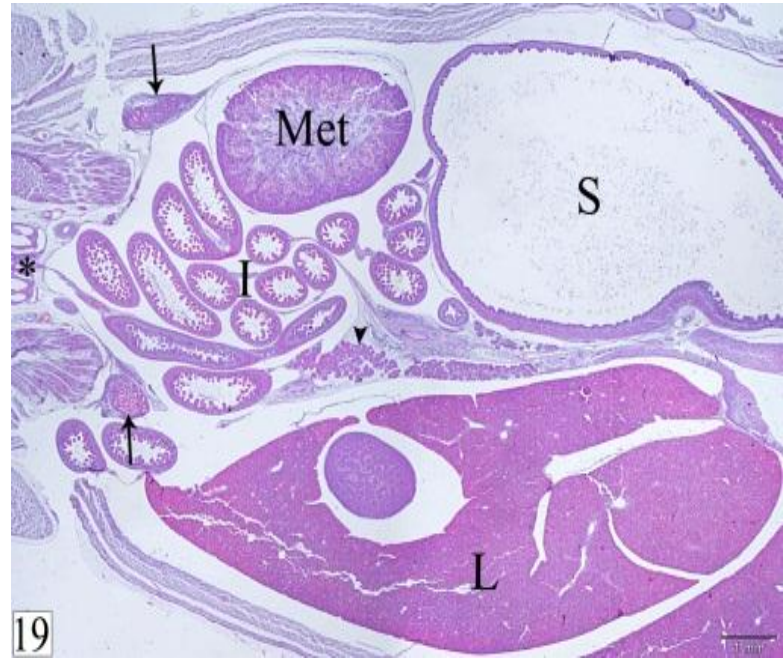

Fig. 19. Frontal section in a rabbit embryo at E 23 showing remnants of the mesonephros (arrows) just cranial to the pelvic inlet (*). Metanephros (Met), intestine (I), liver (L), pancreas (arrow head) and stomach (S). (H\&E, X 12.5). 


\section{DISCUSSION}

The present study shows that the mesonephros of rabbit is a large and long organ extending from the level of the first thoracic somite to the sixth lumbar somite but in human, its extension is restricted where it extends from upper thoracic to upper lumbar (L3) somite (Sadler, 2000). In rabbit, the mesonephros presents in a fully developed state for about 4 days (from 13 to 16 days of gestation). Its degeneration begins early at the $16^{\text {th }}$ day of gestation $(43 \mathrm{~mm})$. These findings are in accordance with many authors.

The relative size of the mesonephros differs in different types of mammals. The pig and rabbit have large mesonephros but sheep has medium size. On the other hand, cat, man and guinea pig have small mesonephros while, mouse and rat practically none at all (Bremer, 1916). It is a mistake to suppose that the larger the mesonephros, the longer it would remain active. The pig has a large mesonephros but it begins to show mesonephric degeneration late. On the contrary, inspite of the rabbit has a large mesonephros, degeneration begins early at about 20 $\mathrm{mm}$ stage. The mesonephros of sheep and cat retain for a long time while, that of man and guinea pig degenerates early (Stanier, 1960).

The current study reveals that the mesonephros in rabbit begins to degenerate early before the metanephros reaches its functional activity i.e. the functions of the mesonephros and the metanephros do not overlap. These results agree with that of Bremer (1916) who stated that the mammalian embryos could be divided into two groups; the first group includes those which retain functional mesonephros until the metanephros begins to secrete urine as in pig, sheep and cat. The other group comprises embryos in which the mesonephros degenerates before the metanephros functions as rabbit, guinea pig, man and rat. The same author added that, in those animals with an early degenerated mesonephros, the placenta is provided with thin plates of epithelium overlying the fetal capillaries and these appear in the placenta at about the time when the mesonephros begin to degenerate. In the placentae of those animals in which the metanephros overlaps the mesonephros, similar plates are not found. On the contrary, Leeson and Baxter (1957) mentioned that, in the rabbit the metanephros overlaps the mesonephros in capability of function. From the abovementioned discussion we believe that, as the mesonephros of the rabbit degenerates early, near the middle of pregnancy, its conjugate placenta modifies into thin plates of epithelium overlying the fetal capillaries at the same time of mesonephric degeneration.

The allantois receives the urine formed within the body of the embryo. There is a close relationship between the size and duration of the mesonephros and the size of the allantois. Those animals with large and long-lasting mesonephros like pig, sheep and cat, have a large sized allantois while, in those with small and early degenerating mesonephros as man and guinea pig, no hollow allantois is formed, only the slender allantoic stalk is found. The rabbit being in the middle with large and early degenerating mesonephros has a medium sized allantois (Bremer, 1916; Stanier, 1960). These results are acceptable for us as our study shows that, the rabbit has a large and early degenerating mesonephros.

Concerning the development and structure of the mesonephros, the current workshows that, the mesonephros generally consists of mesonephric duct, tubules and glomeruli. At E10, the mesonephros consists of mesonephric duct laterally and mesonephric tubules medially. No glomeruli are formed yet. The mesonephric tubules could be differentiated into secretory and collecting parts morphologically and by its position. The secretory tubules are larger, lined with higher epithelium and medially located. However, Leeson and Baxter (1957) in rabbit stated that, in 11-day embryos, the tubules show no morphological differentiation into segments but, at 13-day embryos, morphological and histochemical distinction between proximal and distal segments could be noticed.

In our study, at E 11, the mesonephric corpuscles could be observed for the first time. It consists of glomerulus and glomerular capsule. Some of these glomeruli contain red blood cells. The capsular cavity shows different degrees of its width.

The parietal layer of the glomerular capsule is lined by simple squamous epithelium along the whole length of the mesonephros at different stages of development while, the epithelium of the visceral layer depends on the degree and stage of development of the mesonephros. At E11, in the cranial third of the mesonephros, it is cuboidal epithelium while in the caudal two thirds, it is low columnar. At E 12, it is cuboidal epithelium in the cranial two thirds of the mesonephros while, that of the caudal third remains low columnar epithelium. At E 13, the visceral layer of the glomerular capsule is cuboidal throughout the length of the mesonephros and at E 15, it becomes fully differentiated. In this respect, Leeson and Baxter (1957) in 11-day rabbit embryos indicated that the visceral epithelium of the glomerular capsule is palisade in type while, Osman et al. (2008) in $8 \mathrm{~mm}$ CVRL camel embryos mentioned that the epithelium is flattened.

At E 13, we found that the mesonephros becomes larger than the previous ages therefore, it gets in contact with the surrounding organs. It is related ventrally to the stomach on the left and the liver on the right side. Ventromedially, it is related to the 
gonad. The glomeruli become larger and the intertubular mesenchyme becomes narrow, dense and more vascular.

According to the present article, at E 14\&15 (34\&39 $\mathrm{mm})$, the mesonephros reaches its maximum size and degree of differentiation. All the mesonephric corpuscles and tubules are fully developed. The glomeruli occupy about the medial half of the mesonephros. The mesonephric tubules and duct contain structureless material, the secretory tubules show intense PAS reaction and the intertubular connective tissue becomes scanty. At E 14, the caudal end of the mesonephros is related dorsally to the metanephros but, as a result of the migration of the metanephros cranially, at E 15 , just caudal to its middle, the mesonephros is related dorsally to the metanephros. The right metanephros migrates more cranial than the left one therefore; the accompanied right mesonephros is located somewhat cranial to the level of the left mesonephros.

According to Tiedmann and Wettstein (1980), the mesonephros reaches its maximum size somewhat later (18 days intrauterine life) than that of the present work. In other domestic animals, the mesonephros gains its maximum size at $60 \mathrm{~mm}$ CVRL in pig (Patten, 1964), at 6-8 weeks intrauterine life in bovine (Canfield, 1980), at $32 \mathrm{~mm}$ CVRL in buffalo (Moustafa et al., 1986), at $35 \mathrm{~mm} \mathrm{CVRL}$ in camel (Bareedy et al., 1982 and Osman et al., 2008) and in the middle of the second month in human embryo (Sadler, 2000).

Although the present study reveals that the mesonephros in rabbit reaches its full maturity at $\mathrm{E}$ 15, El-Gharbawy (2002) recorded that at E15, the rabbit mesonephros begins to degenerate. According to our findings, the mesonephric degeneration in rabbits commences at E 16 (43 $\mathrm{mm})$ whereas, Leeson and Baxter (1957) stated that it begins at E 17. Regarding the signs of mesonephric degeneration, we are in agreement with Leeson and Baxter (1957) and Fayez et al. (2014). In other species, degeneration of the mesonephros starts at the $8^{\text {th }}$ week of gestation in bovine (Canfield, 1980), at $47 \mathrm{~mm}$ CVRL in buffalo (Moustafa et al., 1986), at 38, 47 or $150 \mathrm{~mm} \mathrm{CVRL}$ in camel (Emara, 1989; Aly, 2007 and El-Harairy et al., 1998 respectively).

Davies (1952 \& 1953) used the PAS technique to detect protein granules which crossed the glomerular membrane and reabsorbed by the secretory tubules cells and he thought that this is indicative of mesonephric function. In the current work we found that the cells of the secretory tubules begin to show PAS-positive reaction in their luminal border at E 12 and the reaction reaches its maximum at E15 then decreases to be faintly detected in the degenerated tubules. Collecting tubules cells never show PAS activity in the different developmental stages.

\section{CONCLUSION}

The embryogenesis of the rabbit mesonephros passes through different stages which include development, differentiation, function, degeneration and disappearance. It is large, present in fully developed state for about 4 days (from 13 to 16 days of gestation), begins its degeneration early at E 16 and its disappearance starts at E20.

\section{REFERENCES}

Aly, K.H. (2007): Development of the Mesonephros in Camel (Camelusdromedarius). Anat. Histol. Embryol., 36: 58 - 61 .

Arey, L.B. (1965): Developmental anatomy. A text book and laboratory manual of embryology. $7^{\text {th }}$ Ed. W. B. Saunders Co. Philadelphia. London.

Bareedy, M.H.; Anis, H.; Abbas, A.A.; Ewais, M.S.S. and Ammar, S.M.S. (1982): Development of the mesonephros of the dromedary camel. Res. Bull., 498: 1-22.

Bremer, J.L. (1916): The interrelations of the mesonephros, kidney and placenta in different classes of animals. Amer. J. Anat., 19: 179210.

Canfield, P. (1980): Development of the bovine metanephros. Zbl. Vet. Med. C. Anat. Histol.Embryol., 9: 79-107.

Crossmon, G. (1937): A modification of Mallory's connective tissue stain with a discussion of the principals involved. Anat. Rec., 69: 33-38.

Davies, J. (1952): Correlated anatomical and histochemical studies on the mesonephros and placenta of the sheep. Amer. J. Anat., 91: 263300.

Davies, J. (1953): Cytological evidence of protein absorption in fetal and adult mammalian kidneys. Amer. J. Anat., 94: 45-72.

El- Harairy, M.A.; Gaber, A.A. and Attia, K.A. (1998): Prenatal development of the camel (Camelusdromedarius) Kidney: Morphogenesis of mesonephros. Journal of Camel Practice and Research, 5 (1): 115-117.

El-Gharbawy, S.M. (2002): Developmental studies on the kidneys of Rabbits. Vet. Med. J., Giza, 50 (1): 133-155.

Emara, S.A.M. (1989): Prenatal development of the kidney of camel (Camelusdromedarus). M.V.Sc. Thesis. Fac. Vet. Med. Zagazig Univ.

Fayez, S.E.; Sayed-Ahmed, A.; Abo-Ghanema, I.I. and Elnasharty, M.A. (2014): Morphogenesis of rabbit kidney pre-and postnatal. Alexandria Journal of Veterinary Sciences, 41: 47-61.

Harris, H.F. (1900): On the rapid conversion of haematoxylin into haematein in staining reactions. J. Appl. Microsc. Lab. Methods, 3: 777-780. Cited by Bancroft (1996).

Leeson, T.S. and Baxter, J.S. (1957): The correlation of structure and function in the mesonephros 
and metanephros of the rabbit. J. Anat., Lond., 91: 383-390.

McManus, J.F.A. (1946): Histological demonstration of mucin after periodic acid. Nature (London), 158: 202.

Moustafa, I.A.; Enany; E.E.; Osman, A.K. and Amin, M.E. (1986): Development of the mesonephros of the Egyptian water buffalo. Alex. J. Vet. Sci., 2 (1): 1-9.

Osman, A.H.K.; Farouk, S.M.; Eidaroos, H. and Ahmed, A.A.M. (2008): Histogenesis of camel mesonephros. SCVMJ, XIII (2): 377-386.
Patten, B.M. (1964): Foundation of embryology. $2^{\text {nd }}$ Ed. Univ. Michigan Medical School.McGraw-Hill Book Company. New York, San Francisco, London, Toronto.

Sadler, T.W. (2000): Langman's Medical Embryology. $6^{\text {th }}$ Ed., Williams and Wilkins.

Stanier, M.W. (1960): The function of the mammalian mesonephros. J. Physiol., 151: 472-478.

Tiedmann, K. and Wettstein, R. (1980): The mature mesonephric nephron of the rabbit embryo, I. SEM. studies. Cell tissue Res., 209 (1): 95109.

\section{تظور الكلية المتوسطة للأرنب في مرحلة ما قبل الولادة}

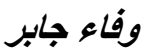

Email: wafaa.anatomy@gmail.com

Assiut University web-site: www.aun.edu.eg

أجري هذا البحث على إحدى وثلاثين جنينا للأرنب نراوحت أعمار هم ما بين اليوم العاثر والرابع والعشرون. وقد أظهرت هذه

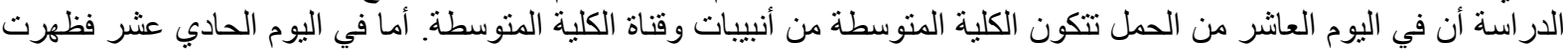

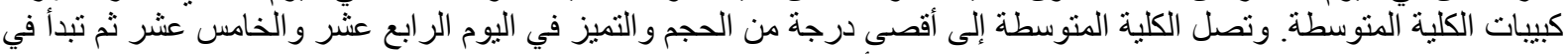

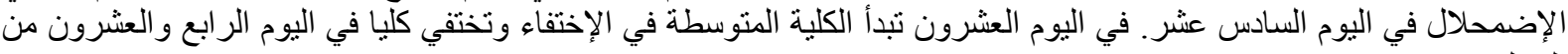

